\title{
Artikelserie zur Geschichte der deutschen Rheumatologie nach dem zweiten Weltkrieg
}

\section{A Series of Articles Concerning the History of German Rheumatology after the Second World War}

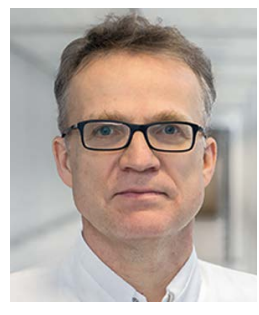

Gernot Keyßer

\author{
Bibliografie \\ Akt Rheumatol 2020; 45: 512 \\ DOI $10.1055 / \mathrm{a}-1283-9009$ \\ ISSN 0341-051X \\ (C) 2020. Thieme. All rights reserved. \\ Georg Thieme Verlag KG, Rüdigerstraße 14, \\ 70469 Stuttgart, Germany \\ Korrespondenzadresse \\ apl. Prof. Dr. med. habil. Gernot Keyßer \\ Leiter des Bereichs Rheumatologie \\ Universitätsklinikum Halle \\ Department für Innere Medizin \\ Klinik für Innere Medizin II \\ Ernst-Grube-Straße 40 \\ 06120 Halle (Saale) \\ Deutschland
}

Liebe Leserinnen und Leser der Aktuellen Rheumatologie

Die Geschichte des Fachgebiets der Rheumatologie beginnt schon mit Hippokrates, der in der Antike die Gicht mit treffenden Beobachtungen charakterisiert hat. Die von ihm propagierte Säftelehre hat letztlich auch den Begriff „Rheumatismus“ geprägt, der „das Fließende “ bedeutet und auf der Annahme beruht, dass krankmachende Säfte vom Körper in die Gelenke abfließen. Eine wissenschaftlich begründete Rheumatologie mit klar voneinander unterscheidbaren Krankheitsbildern, einer hilfreichen Diagnostik und wissenschaftlich geprüften Behandlungsmethoden ist allerdings erst ein Kind des 20. Jahrhunderts. Die politischen Wirren dieser Zeit, 2 Weltkriege, die Teilung Europas in die Länder vor und hinter dem Eisernen Vorhang - all das hat Spuren auch in der Geschichte der Rheumatologie hinterlassen. Manches aus dieser Historie schien vergessen, und wurde erst durch geduldige Recherche wieder ans Tageslicht gebracht. So sei an das Schicksal von Max Hirsch (1875-1941) erinnert, einem der Gründerväter der Deutschen Gesellschaft für Rheumatologie, der als Jude vor den Nazis in die damalige Sowjetunion floh und den antisemitischen Pogromen in Riga zum Opfer fiel [1].

Mit der Teilung Deutschlands nach dem zweiten Weltkrieg ging die deutsche Rheumatologie über Jahrzehnte hinweg getrennte Wege. Augenfällig illustrierte sich diese Trennung darin, dass in der alten Bundesrepublik die rheumatoide Arthritis als „chronische Polyarthritis“ bezeichnet wurde, während der entsprechende Begriff in der ehemaligen DDR „Rheumatoidarthritis“ lautete. Allerdings rissen die kollegialen Kontakte über die Grenze hinweg nie ganz ab, und die Vereinigung der ost- und westdeutschen Fachgesellschaf- ten konnte nach der politischen Wende 1989 auf einem guten Fundament aufbauen. Es lohnt sich grade für jüngere Rheumatologen sehr, Ärztinnen und Ärzten, die große Teile ihres Berufslebens entweder diesseits oder jenseits der innerdeutschen Grenze verbracht haben, nach ihren Erfahrungen mit den unterschiedlichen politischen, wirtschaftlichen und gesellschaftlichen Strukturen zu befragen. Dabei wird immer deutlich werden, dass beide Systeme ihre Vor- und Nachteile hatten und eine geschichtsbewusste Betrachtung die Erfolge beider Gesellschaftsformen würdigen kann.

Mit diesem Heft beginnt eine Serie, bei der Rheumatologen aus der ehemaligen DDR und der „alten“ Bundesrepublik Deutschland ihre Berufserlebnisse schildern. In ihren Berichten spiegeln sich zum einen die globalen Fortschritte der Rheumatologie wider, die mit teilweise unterschiedlichen Tempi in die alltägliche Praxis eingingen. Zusätzlich machen die Beiträge deutlich, was die Rheumatologie in beiden Teilen Deutschlands in den Jahren nach dem Mauerbau trennte und was sie verband.

Wir wünschen Ihnen viel Vergnügen bei der Lektüre. Gernot Keyßer

\section{Literatur}

[1] Keitel W, Olsson L, Matteson EL. Max Hirsch (1875-1941): His forgotten fate and his contributions to the founding of modern rheumatology. Eur J Rheumatol 2016; 3: 101-105. doi: 10.5152/ eurjrheum.2016.15101 